\title{
The Lactic Acid Bacteria in Fermented Durian (D. zibethinus)
}

\section{Hasanuddin}

Faculty of Agriculture, University of Bengkulu

Jl. WR. Supratman Kandang Limun, Bengkulu 38371, Phone 0736-21170 Indonesia

Coresponding author : hasanuddin@unib.ac.id

\begin{abstract}
Lactic acid $(\mathrm{C} 2 \mathrm{H} 5 \mathrm{COOH})$ is the organic acid that can serve as a food preservation. The group of bacteria which can produce lactic acid in their fermentation process known as Lactic Acid Bacteria (LAB). A fundamental biochemical change of fermentation is that an acidic environment is created. Many harmful organisms cannot exist in acidic solution so the fermentation productions are save to eat. Fermented Durian is the fermented food prepared from spontaneous fermentation of durian (Durio zibethinus) with or without salt by wild bacteria. The research was conducted to isolate and identify lactic acid bacteria in tempoyak. The data in this study were laboratory analysis. Samples were collected weekly in a month analyzed microbiologically from traditionl markets in Bengkulu. There were four species of lactic acid bacteria involved in fermented durian namely Leuconostoc mesentroides, Pediococcus acidilactici, Lactobacillus plantarum, and Lactobacillus curvatus.
\end{abstract}

Key words: durian, bacteria, fermentation, lactic acid

Citation to this paper should be made as follows:

Hasanuddin. 2021. The Lactic Acid Bacteria in Fermented Durian (D. zibethinus). Agritropica: Journal of Agricultural Science. 4 (1): 75-81. DOI: https:// doi.org/10.31186/Jagritropica.4.1.75-81.

\section{INTRODUCTION}

Lactic acid bacteria are group of bacteria have their ability to produce lactic acid from substrate of sugars in fermentation process. According to Stamer (1979) the term of lactic acid bacteria is including a group of microorganisms which have their ability to produce lactic acid from carbohydrates resources of their fermentation processes. Lactic acid bacteria involve in many food fermentation products such as vegetables, milk and meat. Furthermore, Dyson and McShane (2009) reported that a fundamental chemical underpinning of fermentation is that an acidic environment is created. Many harmful microorganisms cannot exist in an acidic solution. While lactic acid bacteria go to work on carbohydrates found in flour, grain, dairy products and vegetables to produce an acidic environment that is both suitable for the preservation of the food and for the changing the nature of the flavour of the food. Lactic acid bacteria also can turn the cyanide compounds into cyanic gases which than escape from fermenting food, thus rendering it harmless.

Fermented durian is a kind of traditional spontaneous fermented food prepared from durian (durio zibethinus) by wild microorganisms. Fermented durian is normally prepared from excess, poor quality or over-ripe of durian. The processing of fermented durian is very simple, prepared by mixing the durian pulp with or without salt and left to ferment anaerobic condition at room temperature for week or ten days. 
Contamination with spontaneous wild microorganisms caused biochemical changes of substrate into lactic acid. There are many benefits from food fermentation that make it so widespread in almost every culture throughout the world, high profit from the fermentation procedure is low-cost, low-energy process, and production efficiency in food preservation.

Fermented durian is found not only in Bengkulu but also as long as Sumatera island with different names, and also in Malaysia. Until now, very litle study has been done, recent study reported that the dominant microorganisms in fermented durian was Lachtobacillus plantrum (Leisner et al, 2001). According to Keith (2010) indigenous food fermentations, fermentation in which organic acids are major products, in this category includes Korean kimchi, sauerkraut, fermented mild and cheeses. African ogi and uji, idli, dosai, tape and also tempe, in which acidification occurs during the initial soaking of the soybeans. Meanwhile, according to Leisner et al (2001). fermented durian is a traditional food made from tradision Malaysian durian pulp with or without the addition of salt. Furthermore, Amin et al (2004) reported that in Malaysia fermented durian normally prepared from the poor quality or over-ripe durian with the addition of $1.3 \%$ salt to support the growth of lactic acid bacteria and left to ferment at room temperature for 3-7 days.

The typical processing of durian use the traditional methods are well known as traditional foods. The term of traditional food is food, ingredients, naturally including herbs, food, and beverages traditionally prepared and consumed by local Indonesian community (Dewan Riset Nasional, 1995). Growth and development of commercial food processing industry are caused by several things including: (1). To increase the seasonal level of the availability of fruits. (2) Fermented fruits and vegetables provide a mechanism that causes the fresh ingredients transformed into the components of their respective acid without reducing the nutritional value and physical properties of the products. (3) Can be applied with minimal equipment mechanically and economically so that the processing process can be applied cheaply (Fleming, 1988; Prescott and Dunn, 1958).

\section{MATERIALS AND METHODS}

This research was conducted at Laboratory of Agricultural Technology, Faculty of Agriculture University of Bengkulu. The samples were collected weekly in a month from traditional markets in Bengkulu. The sample was diluted with distilled water with various serial dilutions. Each of the samples was taken $1 \mathrm{ml}$ and cultured on Glucose Yeast Peptone Agar (GYP), and incubated at $35^{\circ} \mathrm{C}$ for 48 hours. The single colony was collected and purified by the method of Streak Plate Technique and was grown on slant agar for further observation. Isolates from the various samples was identified gram reaction, shape, size, and composition of the cell, presence or absence of spores, capsule and flagella, test of temperature, catalyst, $\mathrm{NaCl}$ solution, homo / hetero- fermentation, oxygen requirement, and acid production from various carbon resources. Based on test results, the isolates were grouped into similar characteristics. This identification method refers to methods of Buhanan and Gibbon (1976) and Speck (1976). 


\section{RESULTS AND DISCUSSION}

The observation of cell shape, gram reaction, catalyst, gas from glucose, the ability to live on $15 \% \mathrm{NaCl}$, the ability to live at a temperature of $10^{\circ} \mathrm{C}, 45^{\circ} \mathrm{C}$ and $50^{\circ} \mathrm{C}$ and acid production from various carbon resources, the isolates were classified into 4 groups (Table 1, 2, 3 and 4). Group I represent strains which sphere or ovoid cells in chains, and having cell size ranging 0.9 to 1 micron in diameter. They were gram positive, becoming gram negative with increasing age and acidity. Twenty-four hours cultures to 48 four hours cultures were gram positive and gram negative for 72 hours culture. These homo-fermentative isolates were catalase negative grew best at microaerophilic condition. This group has morphological characteristics such as filliform in stab and slant culture, and irregular surface growth in stab culture, acid on 24 hours lactose agar broth, nonmotile and non-spore formers. The optimum temperture between $10^{\circ}$ to $45^{\circ} \mathrm{C}$. No growth occurred at $50^{\circ} \mathrm{C}$. The strains can produce acid from glucose, sucrose, fructose, lactose, maltose, mannose and arabinose.

Table 1. Characteristics of Pediococcus Isolated from Fermented durian

\begin{tabular}{|c|c|c|c|c|c|c|c|c|c|c|c|c|c|c|}
\hline \multirow{2}{*}{$\begin{array}{l}\text { Isolate } \\
\text { Nos }\end{array}$} & \multirow{2}{*}{$\begin{array}{c}\text { Gram } \\
\text { Reaction }\end{array}$} & \multirow{2}{*}{$\begin{array}{l}\text { Catal } \\
\text { ase }\end{array}$} & \multirow{2}{*}{$\begin{array}{l}\text { Gas from } \\
\text { Glucose }\end{array}$} & \multirow{2}{*}{$\begin{array}{l}15 \% \\
\mathrm{NaCl}\end{array}$} & \multicolumn{3}{|c|}{ Growth at } & \multicolumn{7}{|c|}{ Acid from } \\
\hline & & & & & $10^{\circ} \mathrm{C}$ & $45^{\circ} \mathrm{C}$ & $50^{\circ} \mathrm{C}$ & $\begin{array}{c}\text { Gluc } \\
\text { ose }\end{array}$ & $\begin{array}{c}\text { Sucro } \\
\text { se }\end{array}$ & $\begin{array}{l}\text { Fruc } \\
\text { tose }\end{array}$ & $\begin{array}{l}\text { Lac } \\
\text { tose }\end{array}$ & $\begin{array}{l}\text { Mal } \\
\text { tose }\end{array}$ & $\begin{array}{l}\text { Man } \\
\text { nose }\end{array}$ & $\begin{array}{c}\text { Arabi } \\
\text { nose }\end{array}$ \\
\hline 3. & + & - & - & - & - & + & - & + & + & + & + & + & + & + \\
\hline 5. & + & - & - & - & - & + & - & + & + & + & + & + & + & + \\
\hline 8. & + & - & - & - & - & + & - & + & + & + & + & + & + & + \\
\hline 9. & + & - & - & - & - & + & - & + & + & + & + & + & + & + \\
\hline 10. & + & - & - & - & - & + & - & + & + & + & + & + & + & + \\
\hline 21. & + & - & - & - & - & + & - & + & + & + & + & + & + & + \\
\hline 23. & + & - & - & - & - & + & - & + & + & + & + & + & + & + \\
\hline 25. & + & - & - & - & - & + & - & + & + & + & + & + & + & + \\
\hline 33. & + & - & - & - & - & + & - & + & + & + & + & + & + & + \\
\hline 34. & + & - & - & - & - & + & - & + & + & + & + & + & + & + \\
\hline 36. & + & - & - & - & - & + & - & + & + & + & + & + & + & + \\
\hline 41. & + & - & - & - & - & + & - & + & + & + & + & + & + & + \\
\hline 47. & + & - & - & - & - & + & - & + & + & + & + & + & + & + \\
\hline 49. & + & - & - & - & - & + & - & + & + & + & + & + & + & + \\
\hline 50. & + & - & - & - & - & + & - & + & + & + & + & + & + & + \\
\hline 52. & + & - & - & - & - & + & - & + & + & + & + & + & + & + \\
\hline 54. & + & - & - & - & - & + & - & + & + & + & + & + & + & + \\
\hline 58. & + & - & - & - & - & + & - & + & + & + & + & + & + & + \\
\hline 59. & + & - & - & - & - & + & - & + & + & + & + & + & + & + \\
\hline 68. & + & - & - & - & - & + & - & + & + & + & + & + & + & + \\
\hline 69. & + & - & - & - & - & + & - & + & + & + & + & + & + & + \\
\hline 72. & + & - & - & - & - & + & - & + & + & + & + & + & + & + \\
\hline 73. & + & - & - & - & - & + & - & + & + & + & + & + & + & + \\
\hline 77. & + & - & - & - & - & + & - & + & + & + & + & + & + & + \\
\hline 86. & + & - & - & - & - & + & - & + & + & + & + & + & + & + \\
\hline 87. & + & - & - & - & - & + & - & + & + & + & + & + & + & + \\
\hline 103. & + & - & - & - & - & + & - & + & + & + & + & + & + & + \\
\hline 106. & + & - & - & - & - & + & - & + & + & + & + & + & + & + \\
\hline
\end{tabular}

$+=$ grow $/$ react $-=$ no growth or no reaction. All cultures with cell shape Coccus with size from 0.9 to 1 micron, this culture is the species Pediococcus acidilactici. 
They did not produce gas from glucose and did not grow in $15 \% \mathrm{NaCl}$ solution. Based on these characteristics, the strains which belonged to Group I was Pediococcus acidilactici (Fig.1).

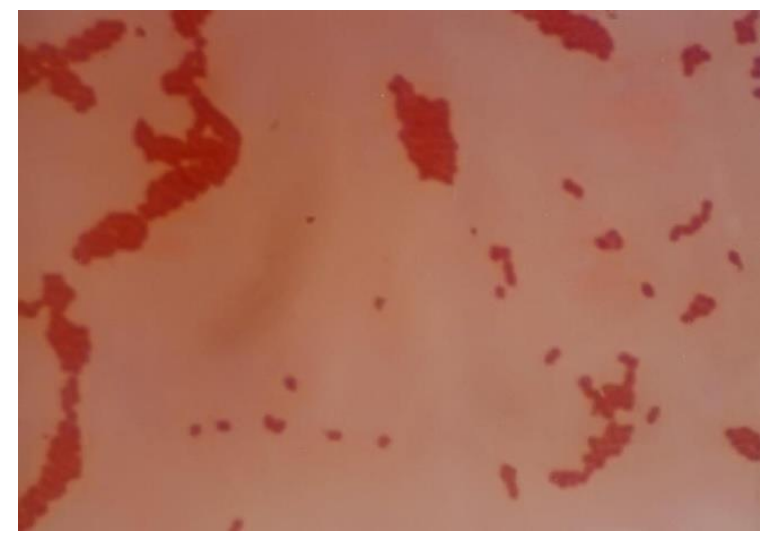

Figure 1. Photo micrographs of bacteria Pediococcus acidilactici isolated from fermented durian
The second group consisted of strains which were gram positive rods less than 5 micron wide with square ends and formed chain. Flagella were absent and non-motile, catalase negative and microaerophilic oxygen requirement. Filliform growth in stab and slant and irregular surface growth on lactose stab culture were observed. Gram positive, became gram negative after 48 hours of growth and with increasing acidity of media. No gas formation was produced from glucose. The homofermentative lactic acid bacteria formed acid from glucose, sucrose, fructose, lactose , maltose, mannose and arabinose. The strains grew in $15 \% \mathrm{NaCl}$ solution and optimum growth between $10^{\circ}$ to $45^{\circ} \mathrm{C}$. Based on these characteristics, the strains which belonged to Group II was Lactobacillus plantarum (Fig.2).

Table 2. Characteristics of Lactobacillus Isolated from fermented durian

\begin{tabular}{|c|c|c|c|c|c|c|c|c|c|c|c|c|c|}
\hline \multirow{2}{*}{$\begin{array}{l}\text { Isolate } \\
\text { Nos }\end{array}$} & \multirow{2}{*}{$\begin{array}{c}\text { Gram } \\
\text { React } \\
\text { ion }\end{array}$} & \multirow[b]{2}{*}{$\begin{array}{l}\text { Catal } \\
\text { ase }\end{array}$} & \multirow{2}{*}{$\begin{array}{l}\text { Gas from } \\
\text { Glucose }\end{array}$} & \multicolumn{3}{|c|}{ Growt at } & \multicolumn{7}{|c|}{ Acid from } \\
\hline & & & & $\begin{array}{c}15 \% \\
\mathrm{NaCl}\end{array}$ & $10^{0} \mathrm{C}$ & $45^{0} \mathrm{C}$ & $\begin{array}{c}\text { Gluco } \\
\text { se }\end{array}$ & $\begin{array}{c}\text { Sucr } \\
\text { ose }\end{array}$ & $\begin{array}{c}\text { Fruc- } \\
\text { tose }\end{array}$ & $\begin{array}{c}\text { Lact } \\
\text { ose }\end{array}$ & $\begin{array}{c}\text { Malt } \\
\text { ose }\end{array}$ & $\begin{array}{c}\text { Mann } \\
\text { ose }\end{array}$ & $\begin{array}{c}\text { Arabi } \\
\text { nose }\end{array}$ \\
\hline 38. & + & - & - & + & - & + & + & + & + & + & + & + & + \\
\hline 43. & + & - & - & + & - & + & + & + & + & + & + & + & + \\
\hline 46. & + & - & - & + & - & + & + & + & + & + & + & + & + \\
\hline 56. & + & - & - & + & - & + & + & + & + & + & + & + & + \\
\hline 57. & + & - & - & + & - & + & + & + & + & + & + & + & + \\
\hline 62. & + & - & - & + & - & + & + & + & + & + & + & + & + \\
\hline 65. & + & - & - & + & - & + & + & + & + & + & + & + & + \\
\hline 66. & + & - & - & + & - & + & + & + & + & + & + & + & + \\
\hline 76. & + & - & - & + & - & + & + & + & + & + & + & + & + \\
\hline 80. & + & - & - & + & - & + & + & + & + & + & + & + & + \\
\hline 81. & + & - & - & + & - & + & + & + & + & + & + & + & + \\
\hline 83. & + & - & - & + & - & + & + & + & + & + & + & + & + \\
\hline 89. & + & - & - & + & - & + & + & + & + & + & + & + & + \\
\hline 92. & + & - & - & + & - & + & + & + & + & + & + & + & + \\
\hline 95. & + & - & - & + & - & + & + & + & + & + & + & + & + \\
\hline 97. & + & - & - & + & - & + & + & + & + & + & + & + & + \\
\hline 100. & + & - & - & + & - & + & + & + & + & + & + & + & + \\
\hline 102. & + & - & - & + & - & + & + & + & + & + & + & + & + \\
\hline
\end{tabular}

$+=$ grow $/$ react $-=$ no growth or no reaction. All cultures with Bacil cell shape with sizes from 1 to $1.2 \times 3-5$ micron, species of this culture is Lactobacillus plantarum 


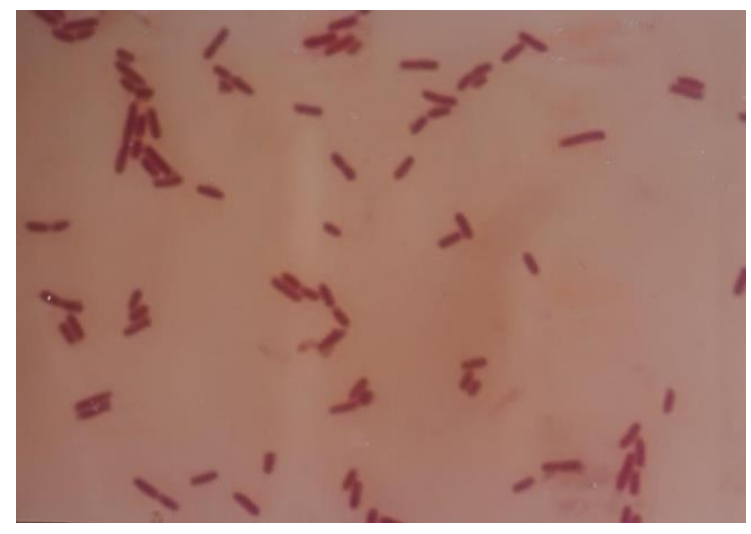

Figure 2. Photo micrographs of Bacteria Lactobacillus plantarum isolated from fermented durian

The characteristics of group III were gram positive rods less than 1.5 micron wide with square ends and formed chain. Flagella were absent and non-motile, catalase negative and microaerophilic oxygen requirement. Filliform growth in stab and slant and irregular surface growth on lactose stab culture were observed. Gram positive, became gram negative after 48 hours of growth and with increasing acidity of media. No gas formation was produced from glucose. The homofermentative lactic acid bacteria formed acid from glucose, sucrose, fructose, lactose , maltose, mannose, but did not form from arabinose. The strains grew in $15 \% \mathrm{NaCl}$ solution and optimum growth between $10^{\circ}$ to $40^{\circ} \mathrm{C}$. Based on these characteristics, the strains which belonged to Group III was Lactobacillus curvatus (Fig. 3).

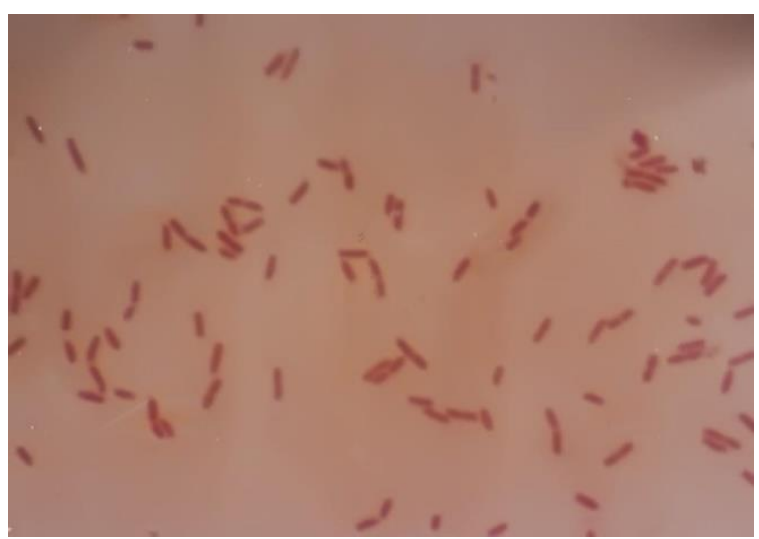

Figure 3. Photo micrographs of Bacteria Lactobacillus curvatus isolated from fermented durian

Table 3. Characteristics of Lactobacillus Isolated from fermented durian

\begin{tabular}{|c|c|c|c|c|c|c|c|c|c|c|c|c|c|}
\hline \multirow{2}{*}{$\begin{array}{l}\text { Iso } \\
\text { late } \\
\text { Nos }\end{array}$} & \multirow{2}{*}{$\begin{array}{c}\text { Gram } \\
\text { React } \\
\text { ion }\end{array}$} & \multirow{2}{*}{$\begin{array}{c}\text { Catal } \\
\text { ase }\end{array}$} & \multirow{2}{*}{$\begin{array}{l}\text { Gas from } \\
\text { Glucose }\end{array}$} & \multicolumn{3}{|c|}{ Growth at } & \multicolumn{7}{|c|}{ Acid from } \\
\hline & & & & $\begin{array}{l}15 \% \\
\mathrm{NaCl} \\
\end{array}$ & $10^{0} \mathrm{C}$ & $45^{0} \mathrm{C}$ & $\begin{array}{c}\text { Gluc } \\
\text { ose }\end{array}$ & $\begin{array}{c}\text { Sucr } \\
\text { ose }\end{array}$ & $\begin{array}{c}\text { Fruc- } \\
\text { tose }\end{array}$ & $\begin{array}{l}\text { Lact } \\
\text { ose }\end{array}$ & $\begin{array}{c}\text { Malt } \\
\text { ose }\end{array}$ & $\begin{array}{l}\text { Man } \\
\text { nose }\end{array}$ & $\begin{array}{l}\text { Arabi } \\
\text { nose }\end{array}$ \\
\hline 7. & + & - & - & + & - & - & + & + & + & + & + & + & - \\
\hline 16. & + & - & - & + & - & - & + & + & + & + & + & + & - \\
\hline 24 & + & - & - & + & - & - & + & + & + & + & + & + & - \\
\hline 27. & + & - & - & + & - & - & + & + & + & + & + & + & - \\
\hline 29. & + & - & - & + & - & - & + & + & + & + & + & + & - \\
\hline 37. & + & - & - & + & - & - & + & + & + & + & + & + & - \\
\hline 40 & + & - & - & + & - & - & + & + & + & + & + & + & - \\
\hline 44. & + & - & - & + & - & - & + & + & + & + & + & + & - \\
\hline 48 & + & - & - & + & - & - & + & + & + & + & + & + & - \\
\hline 51. & + & - & - & + & - & - & + & + & + & + & + & + & - \\
\hline 60. & + & - & - & + & - & - & + & + & + & + & + & + & - \\
\hline 67. & + & - & - & + & - & - & + & + & + & + & + & + & - \\
\hline 78. & + & - & - & + & - & - & + & + & + & + & + & + & - \\
\hline 79. & + & - & - & + & - & - & + & + & + & + & + & + & - \\
\hline
\end{tabular}

$+=$ grow $/$ react $-=$ no growth or no reaction. All cultures with Bacil cell shape with sizes from 0.7 to $0.9 \times 1$ to 1.2 micro, species of this culture is Lactobacillus curvatus. 
Group IV represent strains which sphere or ovoid cells in chains, and having cell size ranging 0.5 to 0.75 micron in diameter. They were gram positive, becoming gram negative with increasing age and acidity. Twenty-four hours culture to 48 were gram positive and gram negative for 72 hours culture. These isolates were catalase negative grew best at microaerophilic condition. This group has morphological characteristics such as filliform in stab and slant culture, and irregular surface growth in stab culture, acid on 24 hours lactose agar broth, nonmotile and non-spore formers. The optimum temperture from $10^{\circ}$ to $45^{\circ} \mathrm{C}$. No growth occured at $50^{\circ} \mathrm{C}$. The strains can produce acid from glucose, sucrose, fructose, lactose, maltose, mannose and arabinose. They produced gas from glucose and grew in $15 \% \mathrm{NaCl}$ solution. Based on these characteristics, the strains which belonged to Group IV was Leuconostoc mesentroides (Fig.4).

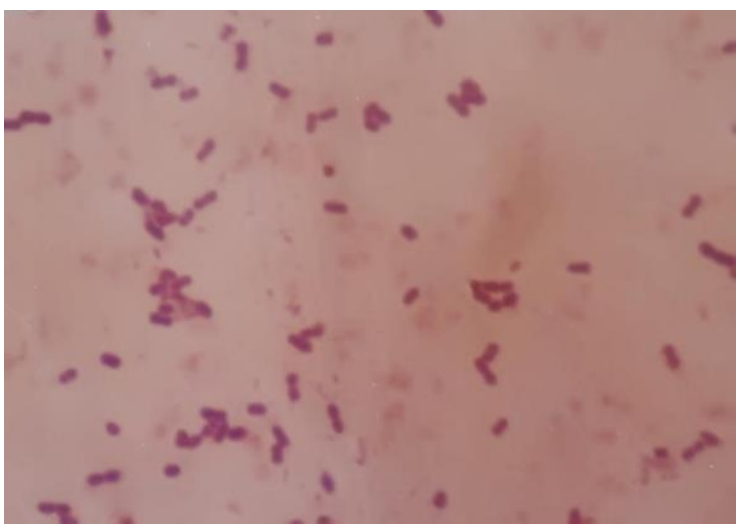

Figure 4. Photo micrographs of Bacteria Leuconostoc mesentroides isolated from fermented durian

According to Rogosa et al (1953) as cited in Bergey's Manual of Determinative Bacteriolgy (1976) the four group strains of isolates were Pediococcus acidilactici, Lactobacillus plantarum, Lactobacillus curvatus and Leuconostoc mesentroides.

Table 4. Characteristics of Leuconostoc Isolated from fermented durian

\begin{tabular}{|c|c|c|c|c|c|c|c|c|c|c|c|c|c|}
\hline \multirow{2}{*}{$\begin{array}{l}\text { Iso- } \\
\text { late } \\
\text { Nos }\end{array}$} & \multirow{2}{*}{$\begin{array}{c}\text { Gram } \\
\text { React } \\
\text { ion }\end{array}$} & \multirow[t]{2}{*}{$\begin{array}{c}\text { Catal } \\
\text { ase }\end{array}$} & \multirow{2}{*}{$\begin{array}{c}\text { Gas } \\
\text { from } \\
\text { Glucose }\end{array}$} & \multicolumn{3}{|c|}{ Growth at } & \multicolumn{7}{|c|}{ Acid from } \\
\hline & & & & $\begin{array}{c}15 \% \\
\mathrm{NaCl}\end{array}$ & $10^{0} \mathrm{C}$ & $45^{0} \mathrm{C}$ & $\begin{array}{c}\text { Gluc } \\
\text { ose }\end{array}$ & $\begin{array}{c}\text { Sucr } \\
\text { ose }\end{array}$ & $\begin{array}{c}\text { Fruc- } \\
\text { tose }\end{array}$ & $\begin{array}{c}\text { Lact } \\
\text { ose }\end{array}$ & $\begin{array}{c}\text { Malto } \\
\text { se }\end{array}$ & $\begin{array}{l}\text { Man } \\
\text { nose }\end{array}$ & $\begin{array}{c}\text { Arabi } \\
\text { nose }\end{array}$ \\
\hline 11. & + & - & + & + & + & + & + & + & + & + & + & + & + \\
\hline 28. & + & - & + & + & + & + & + & + & + & + & + & + & + \\
\hline 30. & + & - & + & + & + & + & + & + & + & + & + & + & + \\
\hline 35. & + & - & + & + & + & + & + & + & + & + & + & + & + \\
\hline 55. & + & - & + & + & + & + & + & + & + & + & + & + & + \\
\hline 61. & + & - & + & + & + & + & + & + & + & + & + & + & + \\
\hline 63. & + & - & + & + & + & + & + & + & + & + & + & + & + \\
\hline 70. & + & - & + & + & + & + & + & + & + & + & + & + & + \\
\hline 82. & + & - & + & + & + & + & + & + & + & + & + & + & + \\
\hline 85. & + & - & + & + & + & + & + & + & + & + & + & + & + \\
\hline 88. & + & - & + & + & + & + & + & + & + & + & + & + & + \\
\hline 91. & + & - & + & + & + & + & + & + & + & + & + & + & + \\
\hline 98. & + & - & + & + & + & + & + & + & + & + & + & + & + \\
\hline
\end{tabular}

$+=$ grow $/$ react $-=$ no growth or no reaction. All cultures with cell shape Coccus with sizes from 0.5 to 0.75 micro level, this culture is Leuconostoc mesentroides. 
While According to Stammer (1979) genus Pediococcus and Leuconostoc are the types of lactic acid bacteria commonly found in fermenting fruits and vegetables.

\section{CONCLUSION}

There werere four species of lactic acid bacteria identified in fermented durian namely: Pediococcus acidilactici, Lactobacillus plantarum, Lactobacillus curvatus and Leuconostoc mesentroides. The four species have positive role in the fermented durian.

\section{REFERENCES}

Amin, A. M., Jaafar, Z., \& Ng, L. K. (2004). Effect of salt on tempoyak fermentation and sensory evaluation. J. Biological Science, 4(5), 650-653.

Buhanan, R. E., \& Gibbons, N. E. (1976). Bergey's Manual of determinative

BacterBacteriology. $8^{\text {th }}$ Ed. Baltimore: The Williams and Wilkins Company, Baltimore.

Dewan Riset Nasional. (1995).

Makanan Tradisional. Widya Karya Nasional Makanan Tradisional 6-8 Juni 1995. Jakarta.

Dyson, S., \& McShane, R. (2009). Fermented Food: The benefits and necessity of fermenting as a process. Food Article, 1-4.
Fleming, H. P. (1988). Fermented Vegetable in Rose, A.H (Ed) Economic Microbiology, Fermented Food. Academic press. New York.

Keith H. Stainkraus (2010). Indigenous Fermented Food Technologies For Small-scale Industries. Institute of Food Science, Cornell University, Geneva, New York, USA.

Leisner, J. J., Vancanneyt, M., Rusul, G., Pot, B., Lefebvre, K., Fresi, A., \& Tee, L. K. (2001). Identification of lactic acid bacteria constituting the predominating microflora in an acid-fermented condiment (tempoyak) popular in Malaysia. International Journal of Food Microbiology,63(1-2), 149-157.

Prescott, S.C \& Dunn. (1958). Industrial Microbiology. 3Ed. Internasional Student Edision. McGrawHill Book Company. New York.

Speck, M. L. (1976). Compedium of Methods for the Microbiological Examination of Food. American Publich Health Association Washington D.C.

Stamer, J. R. (1979). The lactic acid bacteria : Microbes of diversity. Food Technology. I : 60-65 